gene. The HIF-1 is known to bind to hypoxia response elements, which aid in angiogenesis and metastasis.

We know that the tumor stage grade can predict hypoxia. Why is hypoxia important for the oncology community? It is important because it has several implications for imaging and therapy, including radiotherapy and chemotherapy. There have been elegant intratumoral oxygen measurements to correlate this concept of hypoxia and its clinical relevance. One of the newer imaging modalities is the positron emission tomography scan that uses the principle of increased fluorodeoxyglucose by metabolic cancer cells. In hypoxia, this modality can be misleading and warrants use of other 18F- fluoromisnidazole (FIMSA), FAZA, FETA, EF5, and $\mathrm{Cu}$-ATSM that can accurately image even hypoxic tumors. The studies with FIMSA have shown that it can predict survival based on the delineation of the tumor vasculature and hypoxia. Hypoxic cells are resistant to radiotherapy, and several other techniques and the addition of hypoxic sensitizers need to be used concomitantly.

Understanding the molecular biology of the hypoxiainduced substances, we now have engineered molecules such as bevacizumab-a monoclonal antibody to target VEGF. We also have several other monoclonal antibodies and tyrosine kinase inhibitors that target this VEGF pathway and others activated by hypoxia. Thus, an understanding of hypoxia and its consequences is vital to tackle the epidemic of cancer. Although cancer is a genetic disorder involving interplay between dominant oncogenes, tumor suppressor genes, and housekeeping genes, we also understand the profound role hypoxia plays in the subsequent progression and metastasis.

How is this relevant to our community of high altitude hypoxia? We could use the knowledge gained from the oncology community to apply the same principles to fathom high altitude hypoxia. Cross-community collaboration can bring about more meaningful information. As I mentioned earlier, the cancer cell is the most evolved moiety in this universe; we could learn from it its survival tactics and apply them to the larger moiety-human beings-in their quest for adaptation and survival in extremes of conditions.

Govind Babu, MD

Bangalore, India

\section{Polyerythrocythemia and Adaptation to High Altitude}

Chronic mountain sickness (CMS), found at high altitude, is a term that does not explain the etiopathogenesis of the disease in response to the effect of chronic hypoxia. There is no CMS, but rather pulmonary (mainly), cardiac, carotid, kidney, hematological, or genetic disease. All these are associated with an increase in the hematocrit, or what is now known as polyerythrocythemia. Carlos Monge Medrano originally described CMS more than 90 years ago. He was unable to find an explanation for the signs and symptoms and chose to use the term "loss of adaptation." This term was originally accepted, but today it can be appreciated as lacking significance and should stop being used.

Undoubtedly, CMS is a chronic hypoxic process resulting in an increase of hemoglobin due to pulmonary lesions (fundamentally) that alter the pulmonary function, thereby reducing the oxyhemoglobin saturation and stimulating the increase of red blood cells. This occurs, essentially, in pulmonary lesions that are sequelae of diverse lung disease giving rise to intrapulmonary shunts or uneven ventilationperfusion. The term "loss of adaptation" is even semantically inadequate, because in nature, living beings tend to adapt to different environments and circumstances, which could be going to high altitude, temperature changes, solar radiation, ultraviolet radiation, diet changes, and so forth. Consequently, to insist in contemporary medicine on the use of loss of adaptation is not a mistake, but rather, foolishness!

The arterial oxygen content of blood in polyerythrocythemia appears to be increased. However, this apparent increase refers to the maximum oxygen carrying capacity of hemoglobin in blood when fully saturated. That gives rise to two conclusions: 1) that the hemoglobin in patients with polyerythrocythemia tends to be normal and is capable of full saturation when exposed to high oxygen pressures; and 2) in the majority of cases, lung disease that results in intrapulmonary shunts and uneven ventilation-perfusion with reduced alveolar pressure of oxygen or cardiac disease with reduced pulmonary perfusion-both result in the most efficient mechanism to compensate for this ventilatory or cardiac deficiency, namely, polyerythrocythemia.

The misunderstanding of these concepts has led to the wrong interpretation - such as loss of adaptation-of CMS. Throughout history, all living beings go through evolution to adapt to different environmental conditions. Even aging is an evolution that occurs to finish a vital cycle; it never goes in the reverse way. These concepts of CMS and loss of adaptation are unacceptable.

Again, "The organic systems of human beings and all other species tend to adapt to any environmental change and circumstance within an optimal period of time, and never tend towards regression which would inevitably lead to death."

$$
\begin{array}{r}
\text { Gustavo Zubieta-Castillo Sr, MD } \\
\text { Gustavo Zubieta-Calleja Jr, MD } \\
\text { La Paz, Bolivia }
\end{array}
$$

\section{Genome-wide analyses of Tibetan Athletes}

Tibetans have successfully adapted to the hypoxic environment. Especially the high altitude tolerance and athletic ability of Tibetan mountaineers are impressive and fascinating. In order to reveal the genetic basis of hypoxia adaptation and sport ability in these athletes, we performed various types of analyses, including a genome-wide allelic differentiation scan, principal component analysis, Fst and iHS tests, and association study using whole-genome SNP data of Tibetans living in various regions as well as 36 Tibetan mountaineers who successfully reached the top of Mount Everest. A total of 1204 SNPs were selected from the shared 452,456 SNPs in the comparison between non-athlete Tibetans and Han groups by GWADS $(p<0.001)$, of which 121 significant SNPs from 